ARTICLE

https://doi.org/10.1038/s41467-019-09794-7

\title{
Spectra stable blue perovskite light-emitting diodes
}

Yuanzhi Jiang (1D ${ }^{1,6}$, Chaochao Qin²,6, Minghuan Cui ${ }^{2}$, Tingwei He ${ }^{1}$, Kaikai Liu², Yanmin Huang ${ }^{1}$, Menghui Luo ${ }^{1}$, Li Zhang ${ }^{1}$, Hongyu Xu' ${ }^{1}$, Saisai Li ${ }^{1}$, Junli Wei ${ }^{1}$, Zhiyong Liu ${ }^{2}$, Huanhua Wang ${ }^{3}$, Gi-Hwan Kim ${ }^{4}$, Mingjian Yuan ${ }^{1,5}$ \& Jun Chen ${ }^{1,5}$

Device performance and in particular device stability for blue perovskite light-emitting diodes (PeLEDs) remain considerable challenges for the whole community. In this manuscript, we conceive an approach by tuning the 'A-site' cation composition of perovskites to develop blue-emitters. We herein report a Rubidium-Cesium alloyed, quasi-two-dimensional perovskite and demonstrate its great potential for pure-blue PeLED applications. Composition engineering and in-situ passivation are conducted to further improve the material's emission property and stabilities. Consequently, we get a prominent film photoluminescence quantum yield of around $82 \%$ under low excitation density. Encouraged by these findings, we finally achieve a spectra-stable blue PeLED with the peak external quantum efficiency of $1.35 \%$ and a half-lifetime of $14.5 \mathrm{~min}$, representing the most efficient and stable pure-blue PeLEDs reported so far. The strategy is also demonstrated to be able to generate efficient perovskite blue emitters and PeLEDs in the whole blue spectral region (from 454 to $492 \mathrm{~nm}$ ).

\footnotetext{
${ }^{1}$ Key Laboratory of Advanced Energy Materials Chemistry (Ministry of Education), College of Chemistry, Nankai University, 300071 Tianjin, China. ${ }^{2}$ College of Physics and Materials Science, Henan Normal University, 453007 Xinxiang, China. ${ }^{3}$ Institute of High Energy Physics, Chinese Academy of Science, 100049 Beijing, China. ${ }^{4}$ Photonic Energy Research Center, Korea Photonics Technology Institute, 108, Cheomdan venture-ro, Buk-gu, Gwangju 500-779, Republic of Korea. ${ }^{5}$ Renewable Energy Conversion and Storage Center (RECAST), Nankai University, 300071 Tianjin, China. ${ }^{6}$ These authors contributed equally: Yuanzhi Jiang, Chaochao Qin. Correspondence and requests for materials should be addressed to M.Y. (email: yuanmj@nankai.edu.cn)
} 
$\mathrm{P}$ erovskite light-emitting diodes (PeLEDs) attract significant attention because of their high photoluminescence quantum yields (PLQYs), tunable light emission, and high color purity $^{1-10}$. Extensive efforts have been made on improving the device performance of PeLEDs. Very recently, green-emitting, red-emitting, and near-infrared-emitting PeLEDs have obtained impressive external quantum efficiencies (EQEs) exceeding 20\%, which represents an important step toward the commercial application $^{11-13}$. However, blue PeLEDs, as one of the most important prerequisites, tremendously impede the progress in commercialization ${ }^{14-16}$. Device performance, and in particular the device stabilities of blue PeLEDs, remain considerable challenges for the entire PeLED community ${ }^{17-21}$.

In general, two approaches have been developed to obtain blue PeLEDs. The first one relies on incorporating chlorine into bromine-based perovskites to tune the bandgap ${ }^{22-27}$. However, these mixed-halide perovskites exhibit intrinsic phase instabilities, especially under electrical potential ${ }^{20}$, which is induced by the halide migration. Consequently, the electroluminescence (EL) spontaneously transforms from blue- to green color within minutes under device operation ${ }^{22-}$ 24 . The rapid EL degradation has been observed in almost all of the PeLEDs based on 'mixed-halide' strategy ${ }^{28,29}$.

Modulating the quantum-well structure to achieve reduceddimensional (quasi-2D) perovskites represents another effective approach to generate blue PeLEDs ${ }^{2,3}$. By keeping the 'quantum-well' structures stay in an extremely low thickness region $(n \leq 2)$, blue emission can be achieved ${ }^{20-33}$. However, PeLEDs obtained through this strategy commonly exhibit extremely low EQEs $(<0.1 \%)$, together with broad multiple EL peaks, because of the inefficient internal energy transfer. Moreover, Ostwald ripening typically takes place within minutes that leads to EL spectra significantly red shift 16,32 . Thus, this extremely low $n$ values $(n \leq 2)$ quasi-2D perovskites suffer severely from poor spectral stabilities too ${ }^{32}$. Recently, Sargent et al. demonstrate a state-of-the-art sky-blue PeLED following a similar strategy and achieve an EQE of up to $1.5 \%$, together with reasonable spectral stability ${ }^{18}$. However, in their report, the EL spectra cannot reach the real blue region according to the NTSC standard, which impedes their wider applications.

The prior poor device performance and low spectra stabilities for blue PeLEDs are urgently required for a practical remedy. We herein report an approach to engineering 'A-site' cation composition in perovskites to generate blue emitters with good stabilities. We fabricate a Rb-Cs alloyed, $\langle n\rangle_{\mathrm{Rb} 0.6}=$ 3 quasi-2D perovskite and demonstrate its great potential for further pure-blue LED application. Composition engineering and in situ passivation are conducted to further improve its emission property and spectra stabilities. We achieve a prominent film PLQY of around $82 \%$ at $476 \mathrm{~nm}$, with excitation density as low as $1.5 \mathrm{~mW} \mathrm{~cm}^{-2}$. Moreover, no PL spectra shift or broadening have been observed in these materials even after long-time thermal annealing, which demonstrates excellent material stabilities. Eventually, PeLEDs that are displaying pure-blue emission at $475 \mathrm{~nm}$ with a narrow full width at half maximum (FWHM) of around $20 \mathrm{~nm}$, peak brightness of $100.6 \mathrm{~cd} \mathrm{~m}^{-2}$, and peak EQE of $1.35 \%$ are accomplished. Halflifetime $\left(T_{50}\right)$ measurements at the maximum EQE points reveal a lifetime of $14.5 \mathrm{~min}$. These values represent a state-ofthe-art EQE and the highest stability among the blue PeLEDs reported so far' $16,18,22-27,30,31,33-35$. Finally, based on this 'Asite' cation composition engineering, we also demonstrate the full spectrum emission perovskites in the whole blue region (from 454 to $492 \mathrm{~nm}$ ) to meet the various requirements from different industries.

\section{Results}

Properties of 3D Rb-Cs alloyed perovskites. Rubidium $\left(\mathrm{Rb}^{+}\right.$, $152 \mathrm{pm})$ possesses a smaller ionic radius compared with cesium $\left(\mathrm{Cs}^{+}, 167 \mathrm{pm}\right)^{36}$. Thus, incorporating $\mathrm{Rb}^{+}$into $3 \mathrm{D} \mathrm{CsPbBr}_{3}$ perovskites is expected to afford alloyed perovskites, $\mathrm{Rb}_{x}$ $\mathrm{Cs}_{1-x} \mathrm{PbBr}_{3}$, which possess a larger bandgap due to the octahedral titling. $\mathrm{Rb}_{x} \mathrm{Cs}_{1-x} \mathrm{PbBr}_{3}(0 \leq x \leq 1)$ perovskites (Supplementary Fig. 1a) are then fabricated through a solution-based process. As expected, the optical bandgap of $\mathrm{Rb}_{x} \mathrm{Cs}_{1-x} \mathrm{PbBr}_{3}$ films does increase from 2.31 to $2.60 \mathrm{eV}(0 \leq x \leq 0.8)$ with an increasing amount of $\mathrm{Rb}^{+}$(Supplementary Figs. 2a, 29) ${ }^{37}$. However, the stability of $\mathrm{Rb}_{x} \mathrm{Cs}_{1-x} \mathrm{PbBr}_{3}$ declines as the $\mathrm{Rb}^{+}$ratio increases, which ascribes to the increased octahedral distortion caused by continuous $\mathrm{Rb}^{+}$substitution (Supplementary Fig. 2b) ${ }^{37}$. Accordingly, $\mathrm{Rb}_{0.6} \mathrm{Cs}_{0.4} \mathrm{PbBr}_{3}$ is the only stabilized blue emitter among the whole series (Fig. 1c, Supplementary Fig. 3). Unfortunately, $\mathrm{Rb}_{0.6} \mathrm{Cs}_{0.4} \mathrm{PbBr}_{3}$ exhibits a low PLQY of $0.7 \%$ with a fast $\mathrm{PL}$ lifetime of around $0.4 \mathrm{~ns}$, illustrating that a strong trap-mediated non-radiative recombination takes place (Supplementary Fig. 4) ${ }^{10}$. Furthermore, the rough and uneven film morphology is also far from demand (Supplementary Fig. 5).

Quasi-2D structure for Rb-Cs alloyed perovskites. Anyhow, the low PLQY and PL wavelength $(499 \mathrm{~nm})$ diverging from an ideal pure-blue spectral region (from 460 to $480 \mathrm{~nm}$ ) require a solution. As documented, quasi-2D perovskites exhibit much-improved PLQYs and blue-shifted PL emission compared with their 3D analogs, owing to the efficient energy transfer ${ }^{2,3}$. Bearing this in mind, we conceive to fabricate $\langle n\rangle=3 \mathrm{PEA}_{2}\left(\mathrm{Rb}_{x} \mathrm{Cs}_{1-x}\right)_{2} \mathrm{~Pb}_{3} \mathrm{Br}_{10}$ $(0 \leq x \leq 1)$ perovskites with a different $\mathrm{Rb}^{+}$content in order $\mathrm{o}$ obtain efficient blue perovskite emitters (Fig. 1a and Supplementary Fig. 1b). Multiple excitonic absorption peaks are observed in the absorption spectra of $\langle n\rangle=3 \mathrm{PEA}_{2}\left(\mathrm{Rb}_{x} \mathrm{Cs}_{1-x}\right)_{2} \mathrm{~Pb}_{3} \mathrm{Br}_{10}$ $(0 \leq x \leq 1)$, corresponding to the perovskite species with different $n$ values (Fig. 1b) (note: we use ' $\langle n\rangle$ ' that represents a quasi-2D perovskite domain but ' $n$ ' stands for the species with a fixed composition). These characteristics are consistent with previous reports, confirming that quasi-2D perovskites contain a variety of $n$ species rather than a single component ${ }^{2,38}$. Meanwhile, XRD patterns exhibit different diffraction peaks compared with their $3 \mathrm{D}$ analogs caused by the layered structure (Supplementary Figs. 6, 7c, 8 and Supplementary Table 2$)^{39}$.

As expected, PL spectra for pristine $\langle n\rangle=3$ perovskites are shown in Fig. 1c, which do exhibit a significant blue shift compared with their 3D counterparts (Supplementary Fig. 29) 2,40. For instance, the pristine $\langle n\rangle=3 \mathrm{PEA}_{2}\left(\mathrm{Rb}_{0.6} \mathrm{Cs}_{0.4}\right)_{2} \mathrm{~Pb}_{3} \mathrm{Br}_{10}$ (denoted as $\left.\langle n\rangle_{\mathrm{Rb} 0.6}=3\right)$ perovskite displays a single $\mathrm{PL}$ peak at $466 \mathrm{~nm}$ with a FWHM of around $25 \mathrm{~nm}$. In particular, significant PL enhancement (more than 10-fold) is observed for the pristine $\langle n\rangle_{\mathrm{Rb} 0.6}=3$ perovskite when compared with its 3D analog (Fig. 1c), and herein, a PLQY of $11 \%$ is obtained with excitation density as low as 1.5 $\mathrm{mW} \mathrm{cm}{ }^{-2}$. Meanwhile, we also systematically investigate the emission property of the quasi-2D $\mathrm{PEA}_{2}\left(\mathrm{Rb}_{0.6} \mathrm{Cs}_{0.4}\right)_{n-1} \mathrm{~Pb}_{n} \mathrm{Br}_{3 n+1}$ perovskite with the other $\langle n\rangle$ values, and confirm that it is not suitable for further blue PeLED application (Supplementary Figs. 7, 9, 10 and Supplementary Notes 1,2). Even though the PLQY for pristine $\langle n\rangle_{\mathrm{Rb} 0.6}=3$ reaches $11 \%$, it is still far inferior to meet the requirement for PeLED application; thus, further optimization needs to be conducted.

Optical properties of $\mathrm{RbBr}$-rich quasi-2D perovskites. It is documented that increased molar proportion of methylammonium bromide (MABr) in $\mathrm{MAPbBr}_{3}$ could prevent the formation of metallic lead, and further passivate the grain boundary, leading to greatly improved PLQYs ${ }^{1,32}$. Taking this into consideration, we 
a

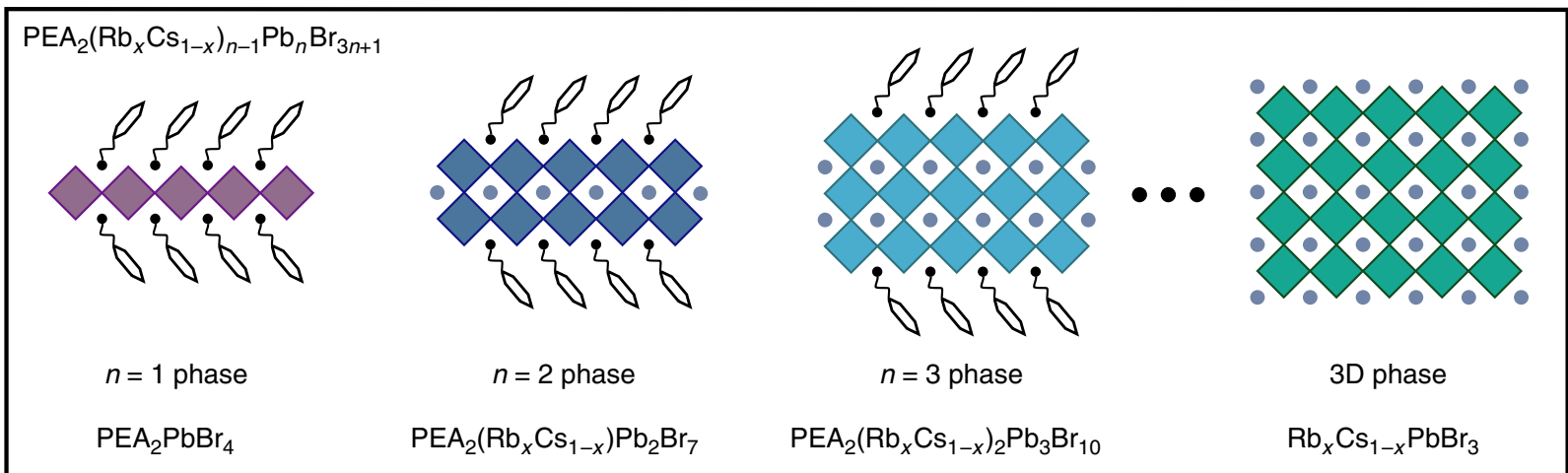

b

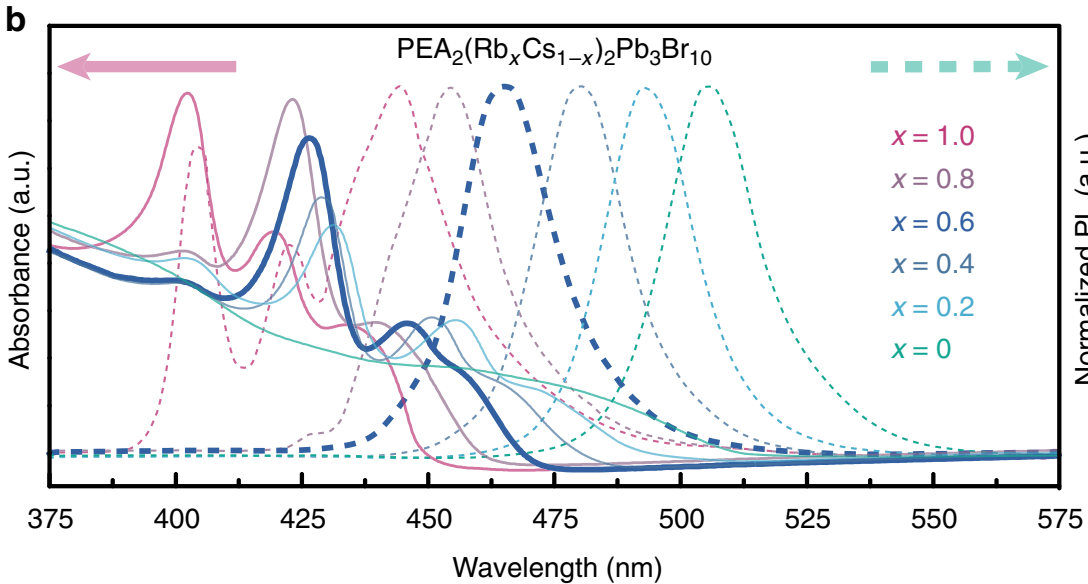

d

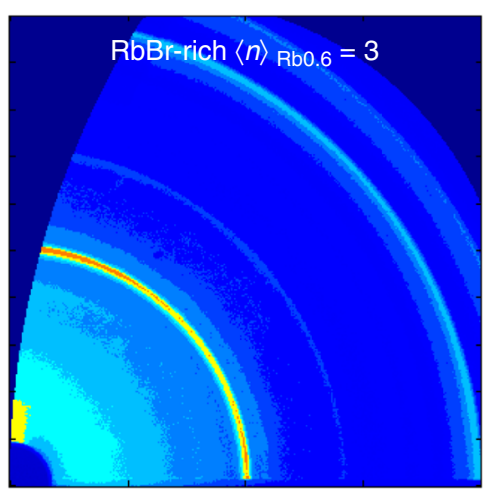

e

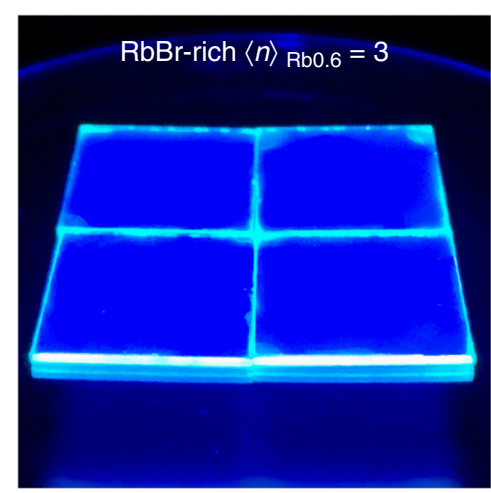

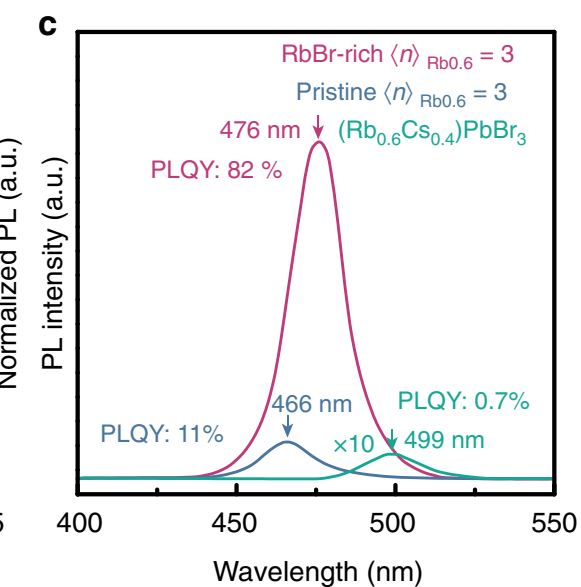

f

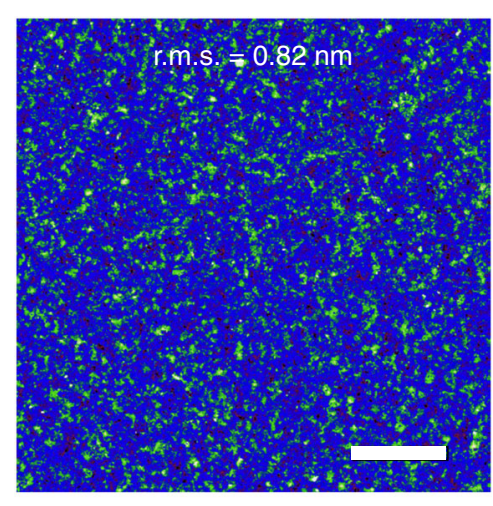

$3.3 \mathrm{~nm}$

$-5.0 \mathrm{~nm}$

Fig. 1 Characteristics of perovskite films. a Cartoon structure of $\mathrm{Cs}-\mathrm{Rb}$ alloyed quasi-2D $P E A_{2}\left(\mathrm{Rb}_{x} \mathrm{Cs}_{1-x}\right)_{n-1} \mathrm{~Pb}_{n} \mathrm{Br}_{3 n+1}$ perovskites. b UV-vis absorption and steady-state $\mathrm{PL}$ spectra of pristine $\langle n\rangle=3 \mathrm{PEA}_{2}\left(\mathrm{Rb}_{x} \mathrm{Cs}_{1-x}\right)_{2} \mathrm{~Pb}_{3} \mathrm{Br}_{10}(0 \leq x \leq 1)$ perovskites. c Steady-state PL spectra and PLQYs of $3 \mathrm{D}$ perovskite $\mathrm{Rb}_{0.6} \mathrm{Cs}_{0.4} \mathrm{PbBr}_{3}$, pristine, and $\mathrm{RbBr}$-rich $\langle n\rangle_{\mathrm{Rb} 0.6}=3$ films. d GIWAXS pattern of the RbBr-rich $\langle n\rangle_{\mathrm{Rb} 0.6}=3$ film. e Photoluminescence image of $\mathrm{RbBr}$-rich $\langle n\rangle_{\mathrm{Rb} .6}=3$ films under excitation. $\mathbf{f}$ AFM height image of RbBr-rich $\langle n\rangle_{\mathrm{RbO} .6}=3$ film with a scale bar of $2 \mu \mathrm{m}$

thus conceive to incorporate an excess amount of $\mathrm{RbBr}$ into the pristine $\langle n\rangle_{\mathrm{Rb} 0.6}=3$ perovskite to form the $\mathrm{RbBr}$-rich $\langle n\rangle_{\mathrm{Rb} 0.6}=3$ perovskite and then investigate its emission property. Meanwhile, excessive $\mathrm{CsBr}$ could essentially induce a cation exchange, leading to a green emitter, which is not interested in this research (Supplementary Fig. 11). As shown, XRD patterns confirm that the $\mathrm{RbBr}$-rich $\langle n\rangle_{\mathrm{Rb} 0.6}=3$ perovskite possesses a very similar diffraction pattern as the pristine one (Supplementary Fig. 12), and no extra RbBr peaks can be detected (Supplementary Fig. 13). Strikingly, we find that the PLQY of $\mathrm{RbBr}-\operatorname{rich}\langle n\rangle_{\mathrm{Rb} 0.6}=3$ perovskite soars up to $82 \%$ at the same excitation density $(1.5 \mathrm{~mW}$ $\mathrm{cm}^{-2}$ ) with an excellent stability (Supplementary Figs. 14, 15, 28, and 30). Moreover, a $10-\mathrm{nm}$ red shift in PL spectra compared with the pristine $\langle n\rangle_{\mathrm{Rb} 0.6}=3$ perovskite is observed (Fig. 1c, Supplementary Figs. 16d, 28). Notably, an immoderate amount of excess $\mathrm{RbBr}$ should be avoided, or it could induce a serious defect state, as well as an irreversible morphology destruction (Supplementary Figs. 17, 18).

Photocarrier transportation and recombination dynamics. Transient absorption (TA) measurement is conducted to investigate the transfer and recombination dynamics of photogenerated carriers in pristine and $\mathrm{RbBr}$-rich $\langle n\rangle_{\mathrm{Rb} 0.6}=3$ quasi- $2 \mathrm{D}$ perovskites. As shown in Fig. $2 \mathrm{a}$ and $\mathrm{b}$, three distinctive groundstate bleach (GSB) peaks are observed in the pristine $\langle n\rangle_{\mathrm{Rb} 0.6}=3$ perovskite. TA spectra at different selected delay times are 

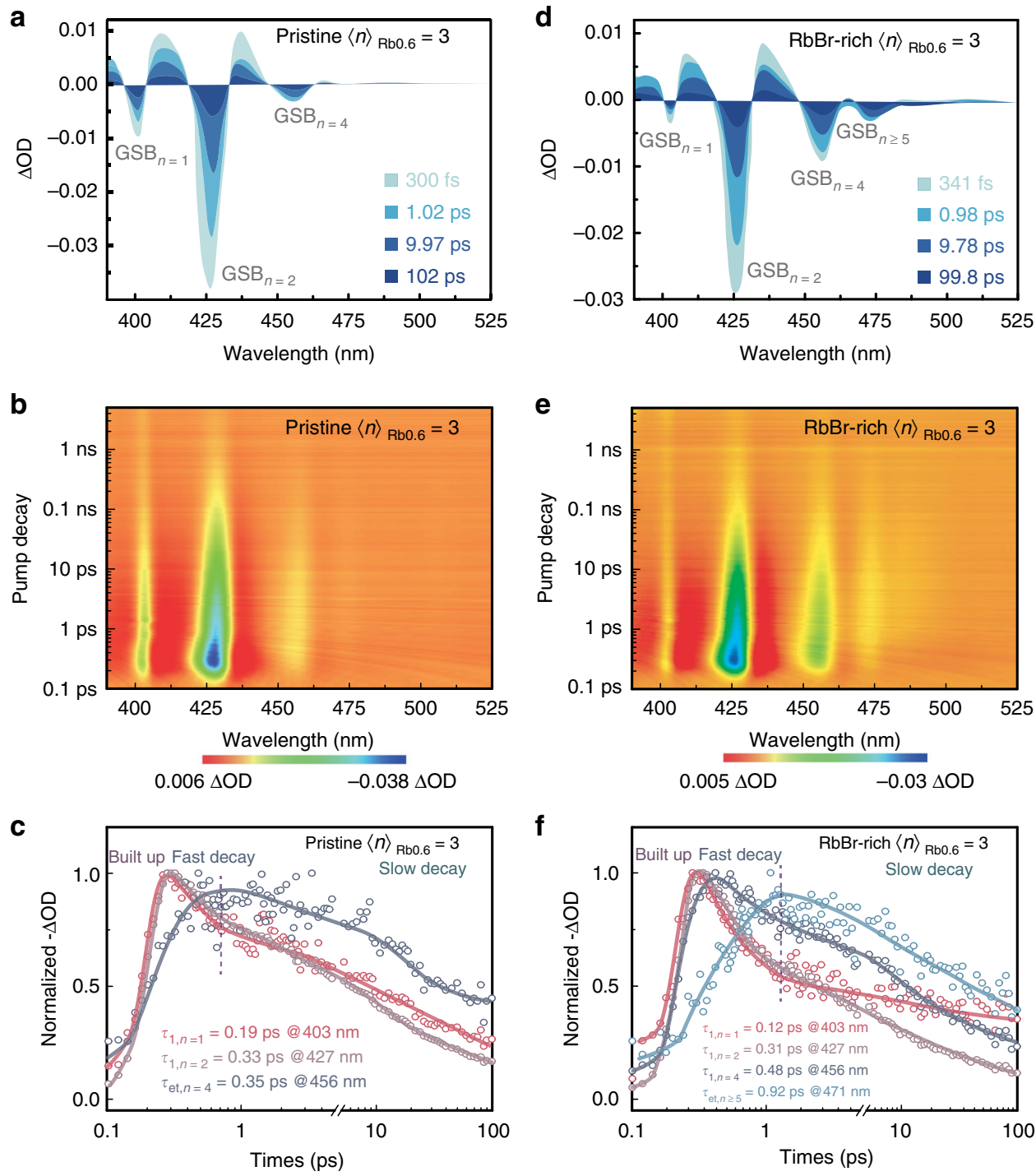

Fig. 2 TA measurements for quasi-2D perovskite films. a, d TA spectra at selected timescales; $\mathbf{b}$, e time-wavelength-dependent TA color maps; and c, f TA spectra at a different wavelength as a function of delay time for pristine and $\mathrm{RbBr}$-rich $\langle n\rangle_{\mathrm{Rb} 0.6}=3$ films, respectively

displayed in Fig. 2a. It is clear that photogenerated excitons are initially formed at $n=1$ and $n=2$ species, and the $\mathrm{GSB}_{n}=4$ gradually emerges with increasing the decay time, indicating that an energy transfer process takes place ${ }^{2,6,41}$. Notably, the GSB of $n=3$ species could not be obviously distinguished, probably due to the closely packed GSB peaks and strong excited state absorption (ESA) signals (Supplementary Figs. 19, 20 and Supplementary Note 3 ). The decay kinetics of each GSB is extracted in Fig. 2c. The fast decay components for $n=1$ and 2 bleaching are ascribed to the carrier transfer from lower- $n$ species (donor domain) to the emitting domain ( $n=4$ species); the slow decay component shall refer to the charge trapping according to previous reports ${ }^{42}$. The fast decay component reveals time constants $\left(\tau_{1}\right)$ of $0.19(n=1)$ and $0.33 \mathrm{ps}(n=2)$, respectively. Coincidentally, the fitted time constant $\left(\tau_{\mathrm{et}}\right)$ of the rising component for $\mathrm{GSB}_{n}=4$ is $0.35 \mathrm{ps}$, which is in good agreement with a fast decay timescale. Accordingly, we can conclude that carrier transfer from donor domains $(n=1$ and 2$)$ to the emitting domain $(n=4)$ is accomplished in picoseconds with near-unit efficiency.

TA characterization for the $\mathrm{RbBr}$-rich $\langle n\rangle_{\mathrm{Rb} 0.6}=3$ perovskite is shown in Fig. 2d, e. Notably, a new GSB peak domain at $471 \mathrm{~nm}$ referring to $n=5$ and $n>5$ species gradually appears, which cannot be observed in the pristine $\langle n\rangle_{\mathrm{Rb} 0.6}=3$ perovskite. The TA data suggest that new perovskite domains, $n=5$ and $n>$ 5 species, appear when introducing an excess of $\mathrm{RbBr}$ into pristine $\langle n\rangle_{\mathrm{Rb} 0.6}=3$. The decay kinetics for different GSB peaks correlate well with the characteristics of energy transfer (Fig. $2 \mathrm{f}$ and Supplementary Table 3 ). In addition, the population in $n=1$, 2 , and 4 species decays faster than carrier injection speed, and the decay kinetics also exhibit a slower lifetime for the lower bandgap species. The GSB maximum sequentially delays for the lower bandgap species $(n \geq 5)$, proving that lower bandgap domains have received carriers from the larger bandgap species. All these details together confirm that carriers have transferred from a wider to a lower bandgap species, and eventually recombined in the $n>5$ domain $^{42,43}$. This more-graded energy landscape leads to a more efficient energy transfer compared with the case of pristine $\langle n\rangle_{\mathrm{Rb} 0.6}=3$ that facilitates the radiative recombination.

Time-resolved PL (TRPL) analysis is in excellent agreement with the TA kinetics (Fig. 3a, b). The PL maximum of pristine $\langle n\rangle_{\mathrm{Rb} 0.6}=3$ perovskite moves from 466 to $476 \mathrm{~nm}$ after the $\mathrm{RbBr}$ rich $\langle n\rangle_{\mathrm{Rb} 0.6}=3$ perovskite formed. We cannot extract the PL lifetime for each higher-bandgap species $(n=1,2$, and 4$)$ to illustrate energy transfer kinetics because of the instrument limitation. The overall PL lifetime for $\mathrm{RbBr}$-rich $\langle n\rangle_{\mathrm{Rb} 0.6}=3$ 

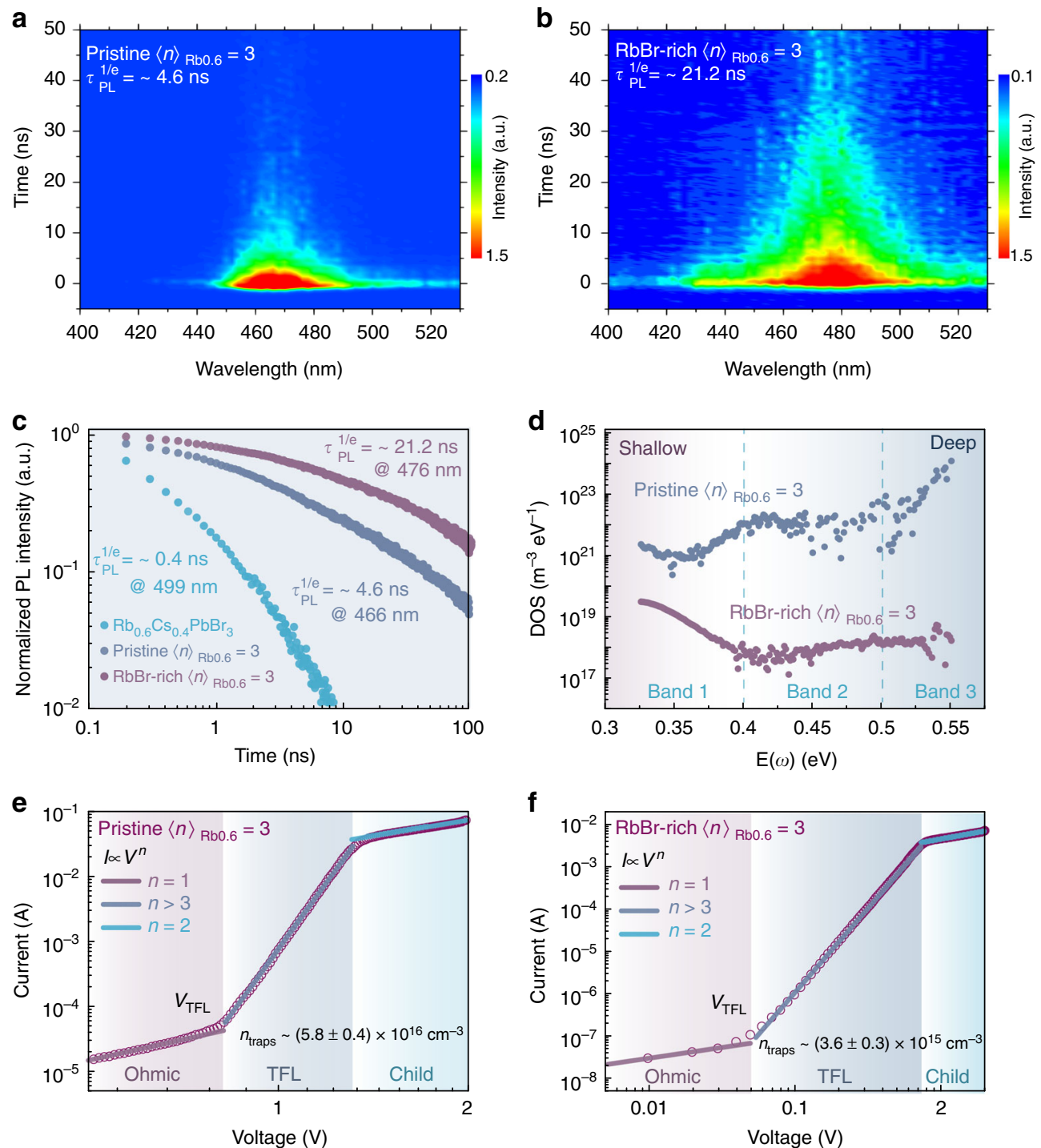

Fig. 3 Characteristics of trap densities. TPRL measurements of (a) pristine and (b) $\mathrm{RbBr}$-rich $\langle n\rangle_{\mathrm{Rb} 0.6}=3$ films. c The $\mathrm{PL}$ lifetime for $3 \mathrm{D} \mathrm{Rb}_{0.6} \mathrm{Cs}_{0.4} \mathrm{PbBr}_{3}$, pristine, and $\mathrm{RbBr}$-rich $\langle n\rangle_{\mathrm{RbO} .6}=3$ films. $\mathbf{d}$ Trap density of states $(\mathrm{tDOS})$ for pristine and RbBr-rich $\langle n\rangle_{\mathrm{RbO} .6}=3$ perovskite in TAS analysis. Current-voltage $(J-V)$ response of $(\mathbf{e})$ pristine and $(\mathbf{f}) \mathrm{RbBr}$-rich $\langle n\rangle_{\mathrm{RbO} .6}=3$ films in SCLC measurement

perovskite was determined to be $21.2 \mathrm{~ns}$, which is around five times as long as the pristine $\langle n\rangle_{\mathrm{Rb} 0.6}=3$ perovskite's lifetime (4.6 ns) (Fig. 3c). The longer PL lifetime generally indicates a lower defect density, which should account for the primary reason for PLQY enhancement ${ }^{5}$.

Electrical characterization of quasi-2D perovskites. Herein, space-charge-limited current (SCLC) and thermal admittance spectroscopy (TAS) technique are used to quantize the defect density. SCLC measurements are conducted with the device structure of ITO/perovskites/ $\mathrm{MoO}_{3} / \mathrm{Au}$. We then measure the $J-V$ response of the different perovskite films in the SCLC regime (Fig. 3e, f). Three distinct regions clearly identify from the $J-V$ curves. At intermediate voltages, the current injection displays a marked nonlinear increase (set in at $V_{\mathrm{TFL}}=0.82 \mathrm{~V}$ for the pristine one, and $0.05 \mathrm{~V}$ for the $\mathrm{RbBr}$-rich one), and curves then switch to the trap-filled-limit (TFL) region, in which all the trap density levels are continuously filled by the increasing voltage. Thus, the onset voltage $\left(V_{\mathrm{TFL}}\right)$ is proportional to the density of trap states $\left(n_{\text {traps }}\right)$. Consequently, we extract a trap state density of $n_{\text {traps }}=3.6 \times 10^{15} \mathrm{~cm}^{-3}$ for $\mathrm{RbBr}$-rich $\langle n\rangle_{\mathrm{Rb} 0.6}=3$ perovskite, which is more than one order of magnitude lower than the pristine $\langle n\rangle_{\mathrm{Rb} 0.6}=3$ perovskite $\left(n_{\text {traps }}=5.8 \times 10^{16} \mathrm{~cm}^{-3}\right)^{44}$.

Furthermore, we also extract the trap density from TAS analysis. TAS technique is recognized as an effective method for characterizing both shallow and deep defects. In general, the energetic profile of trap density of states ( $t$ DOS) can be derived from the angular frequency-dependent capacitance, where the energetic demarcation $E(\omega)$ is correlated with the trap depth. As revealed in Fig. 3d, the $\mathrm{RbBr}$-rich $\langle n\rangle n_{\mathrm{Rb} 0.6}=3$ perovskite exhibits a much lower $t$ DOS than the pristine one over the whole trap depth. In particular, the $\mathrm{RbBr}-$ rich $\langle n\rangle_{\mathrm{Rb} 0.6}=3$ perovskite displays around four orders of magnitude lower deeper trap density (from 0.50 to $0.55 \mathrm{~V}$ ) than its pristine analog. The marked decreased $t \mathrm{DOS}$ in TAS analysis is consistent with the SCLC data, indicating that $\mathrm{RbBr}$ effectively passivates the defect states, especially deep trap states in the pristine $\langle n\rangle_{\mathrm{Rb} 0.6}=3$ perovskite.

Based on the above optical and electrical analysis, we thus conclude that the excessive amount of $\mathrm{RbBr}$ actually plays dual roles in improving the pristine $\langle n\rangle_{\mathrm{Rb} 0.6}=3$ perovskite's 
a

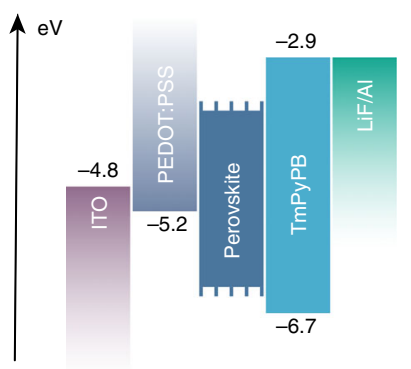

C
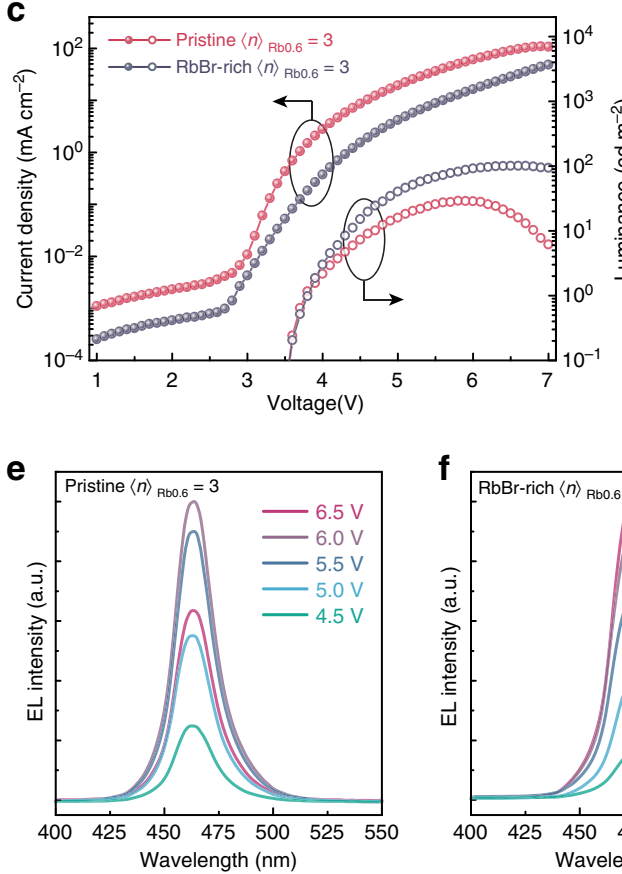

b

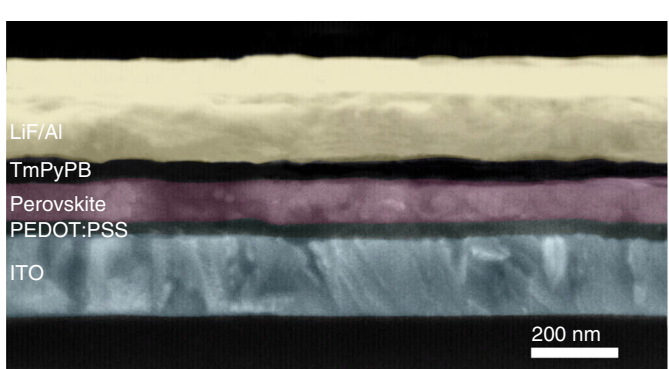

d

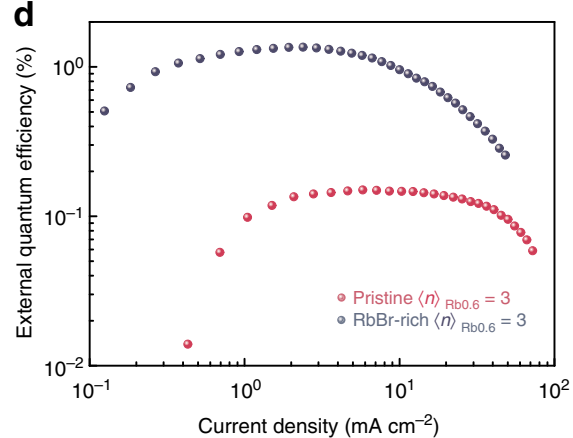

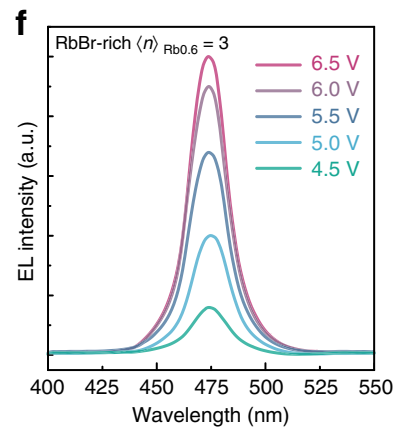

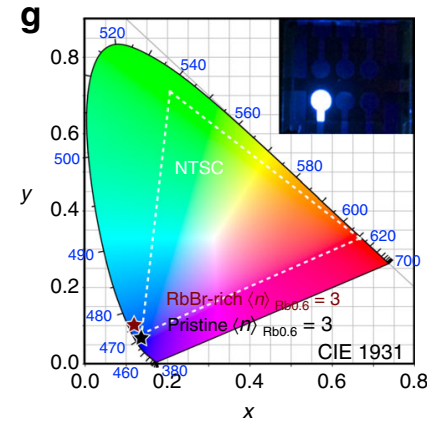

Fig. 4 Optoelectronic characteristics of the PeLEDs. a Band alignment of each function layer in devices. b SEM cross-sectional image of the RbBr-rich $\langle n\rangle_{\mathrm{Rb} 0.6}=3$ based devices. $\mathbf{c} I-V$ and $L-V$ curves for pristine and $\mathrm{RbBr}$-rich $\langle n\rangle_{\mathrm{Rb} 0.6}=3$ devices. $\mathbf{d} \mathrm{EQE}$ characteristics of pristine and $\mathrm{RbBr}-\mathrm{rich}\langle n\rangle_{\mathrm{RbO} .6}=$ 3 devices. EL spectra of (e) pristine and (f) $\mathrm{RbBr}$-rich $\langle n\rangle_{\mathrm{Rb} 0.6}=3$ at different voltage bias. $\mathbf{g}$ The corresponding Commission Internationale de l'Echlaiage (CIE) coordinate of pristine and $\mathrm{RbBr}$-rich $\langle n\rangle_{\mathrm{RbO} .6}=3$ devices, and the inset image shows the electroluminescence of $\mathrm{RbBr}$-rich $\langle n\rangle_{\mathrm{Rb}} .6=3$ based PeLEDs

emission property. First, it induces the $n$-value shift to the larger number and consequently forms new $n=5$ and $n>5$ species. The phenomena are in consistent with previous reports, wherein incorporating an excess of FAI cation in quasi-2D $\mathrm{NMA}_{2} \mathrm{FA}_{n-1} \mathrm{~Pb}_{n} \mathrm{I}_{3 n+1}$ perovskites can induce the $n$ values to slightly shift to the larger number ${ }^{6}$. A more-graded energy landscape is herein formed in the $\mathrm{RbBr}$-rich $\langle n\rangle_{\mathrm{Rb} 0.6}=3$ perovskite $^{41}$. Consequently, funneling carriers into the low concentration $n>5$ subpopulation could facilitate radiative recombination, leading to PLQY enhancement, together with a slightly spectral redshift (around $10 \mathrm{~nm}$ ) (Supplementary Fig. 21). On the other hand, $\mathrm{RbBr}$ effectively passivates the pristine $\langle n\rangle_{\mathrm{Rb} 0.6}=3$ 's defect states, leading to overall several orders of magnitude lower defect density. The dramatically reduced defect density accounts for the primary reason of the PLQY enhancement $^{5}$. Ultimately, the $\mathrm{RbBr}$-rich $\langle n\rangle_{\mathrm{Rb} 0.6}=3$ perovskite film exhibits a prominent PLQY of around $82 \%$ at $476 \mathrm{~nm}$, with low excitation power density of $1.5 \mathrm{~mW} \mathrm{~cm}^{-2}$.

Device structure and performance. Encouraged by the above findings, we further fabricated and optimized the PeLED device using different perovskite films. We configured PeLEDs as the structure glass/ITO/PEDOT:PSS $(30 \mathrm{~nm}) /$ perovskites $(80 \mathrm{~nm}) /$
TmPyPB $(40 \mathrm{~nm}) / \mathrm{LiF}(0.8 \mathrm{~nm}) / \mathrm{Al}(100 \mathrm{~nm})$. The schematic of the band alignment diagram for all function layers is shown in Fig. 4a. The band structure of $\mathrm{RbBr}$-rich $\langle n\rangle_{\mathrm{Rb} 0.6}=3$ film was determined using ultraviolet photoelectron spectroscopy (UPS; Supplementary Fig. 22). The cross-section scanning electron microscopy (SEM) image of the completed device also confirms the stacked film thickness (Fig. 4b).

Compared with the pristine $\langle n\rangle_{\mathrm{Rb} 0.6}=3$ perovskite, an extremely dense and uniform film is observed in SEM images for the $\mathrm{RbBr}$-rich $\langle n\rangle_{\mathrm{Rb} 0.6}=3$ perovskite (Supplementary Fig. 23). As shown in the atomic force microscopy (AFM) image, the surface roughness of the $\mathrm{RbBr}$-rich $\langle n\rangle_{\mathrm{Rb} 0.6}=3$ perovskite film on PEDOT:PSS is only $0.82 \mathrm{~nm}$ (Fig. 1f, Supplementary Fig. 24), which is sufficiently smooth to avoid current leakage. In addition, grazing-incidence wide-angle X-ray scattering (GIWAXS) measurements are conducted. As revealed in Fig. 1d, uniform intensities along the Debye-Scherrer rings are observed, indicating that the quasi-2D perovskite crystallites are isotropically oriented in the film ${ }^{13}$.

Pristine $\langle n\rangle_{\mathrm{Rb} 0.6}=3$ devices exhibit low performance with $0.15 \% \mathrm{EQE}$ and $28.9 \mathrm{~cd} \mathrm{~m}^{-2}$ luminance (Fig. $4 \mathrm{~d}$ ), which is not a surprise in view of its low PLQY. In addition, the device displays a brightness rolloff at around $6 \mathrm{~V}$ (Fig. 4c), indicating that a strong non-radiative recombination takes place. Strikingly, by taking 
advantage of high PLQY and advanced film quality, the RbBr-rich $\langle n\rangle_{\mathrm{Rb} 0.6}=3$ perovskite displays a significant enhancement in EQE of $1.35 \%$, and luminance of $100.6 \mathrm{~cd} \mathrm{~m}^{-2}$ (Fig. $4 \mathrm{~d}$, Supplementary Fig. 25) with good reproducibility (Supplementary Fig. 26). The resulting EL spectra closely match its PL spectra, preserving a narrowband emission. The narrowband emission exhibits excellent color purity with CIE chromatic coordinates of $(0.115,0.099)$ close to NTSC standard values (Fig. 4g), and follows a Lambertian profile (Supplementary Fig. 27). Notably, the result represents the most efficient pureblue PeLED reported so far, which is one-order-of-magnitude enhancement in EQE over the best-reported pure-blue PeLEDs operating in the similar wavelength.

As far as we know, to achieve excellent spectra stability for blue PeLEDs remains a global challenge, owing to the higher driving voltage required. Spectral stability for our pristine and $\mathrm{RbBr}$-rich $<n>_{\mathrm{Rb} 0.6}=3$ PeLEDs is recorded at different bias voltage. The EL spectra as a function of bias voltage are shown in Fig. 4e, f. As bias voltage increased from 4.5 to $6 \mathrm{~V}$, the $\mathrm{EL}$ intensity increased correspondingly, and the EL peak position and FWHM remained unchanged. When the bias voltage further increased up to $6.5 \mathrm{~V}$, the EL intensity of $\mathrm{RbBr}$-rich $\langle n\rangle_{\mathrm{Rb} 0.6}=3$ still increased and exhibited excellent spectral stability. On the other hand, the spectra of pristine $\langle n\rangle_{\mathrm{Rb} 0.6}=3$ also remained unchanged at 6.5 $\mathrm{V}$, in spite of the brightness rolloff. These results undoubtedly demonstrate the excellent spectral stability of the quasi-2D Rb-Cs alloyed perovskites, which have overcome the previous instabilities induced by the halide migration or Ostwald ripening.

Spectral stability of quasi-2D Rb-Cs alloyed perovskites. In order to further demonstrate the spectral stability of the perovskites, the pristine and $\mathrm{RbBr}$-rich $\langle n\rangle_{\mathrm{Rb} 0.6}=3$ perovskite films are first tested under thermal annealing. As shown in Fig. 5a, the
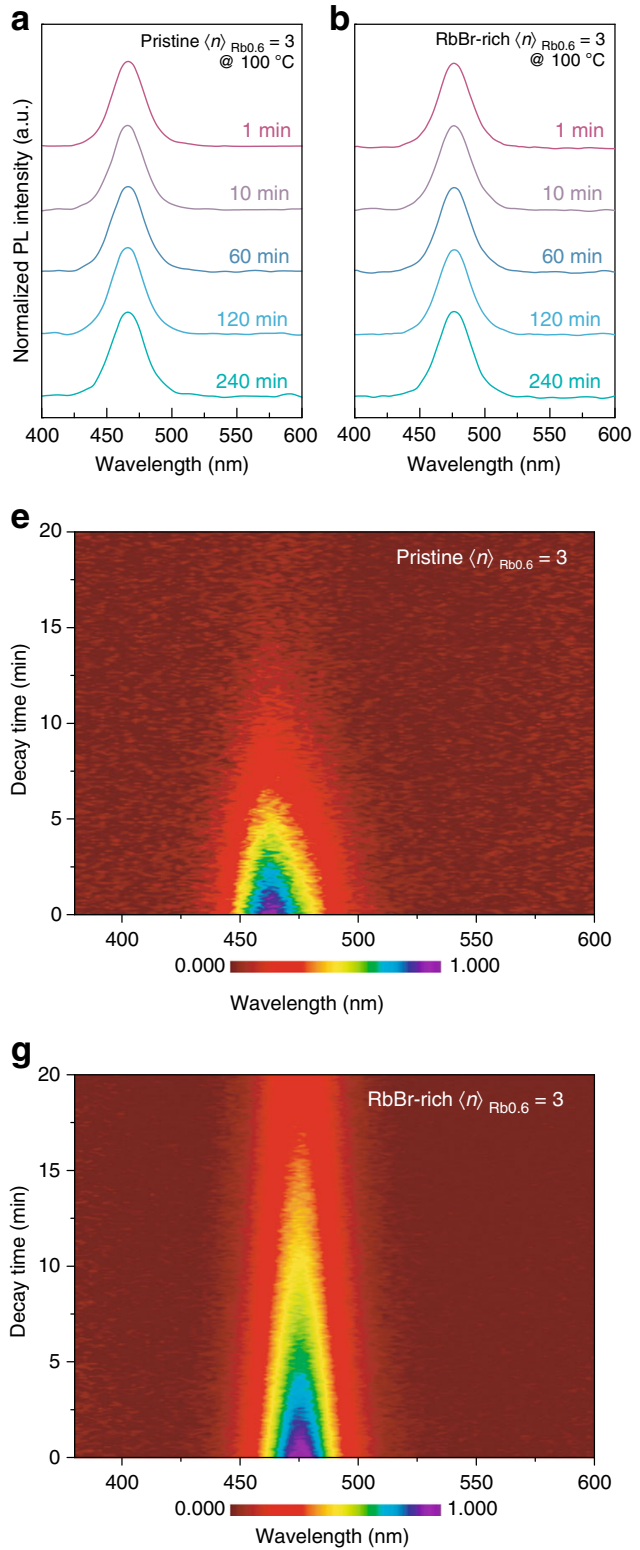
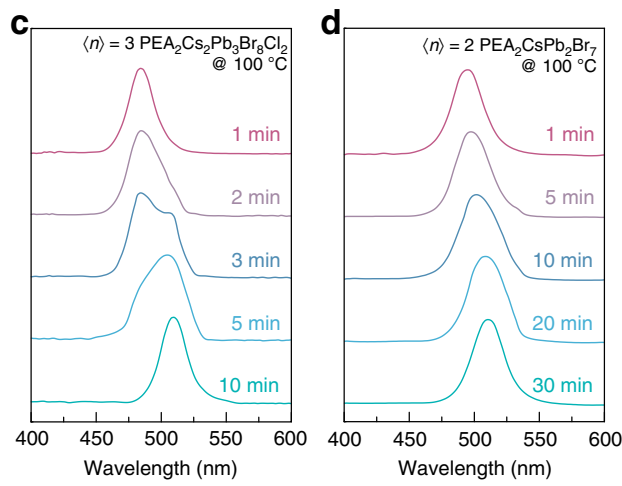

f

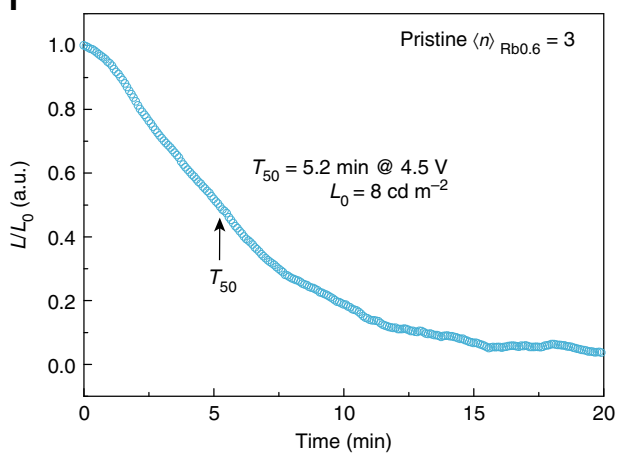

h

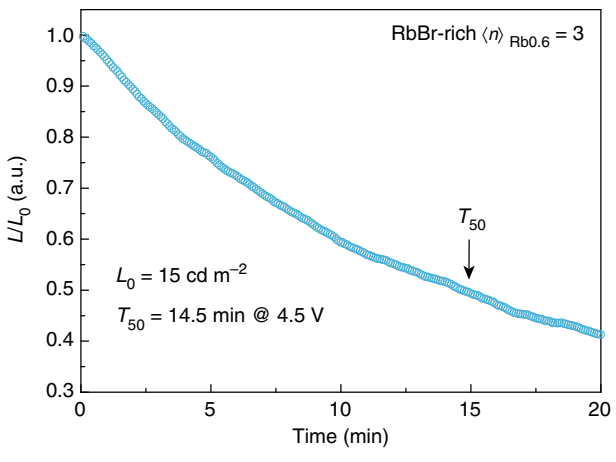

Fig. 5 Spectral stability measurements. PL spectra evolution of (a) pristine $\langle n\rangle_{\mathrm{RbO} .6}=3$, (b) RbBr-rich $\langle n\rangle_{\mathrm{Rbo} .6}=3$, (c) $\langle n\rangle=3$ mixed-halide $\mathrm{PEA}_{2} \mathrm{Cs}_{2} \mathrm{~Pb}_{3} \mathrm{Br}_{8} \mathrm{Cl}_{2}$, and (d) lower-dimensional $\angle n>=2 \mathrm{PEA}_{2} \mathrm{CsPb}_{2} \mathrm{Br}_{7}$ perovskites after continuous thermal treatment $\left(100^{\circ} \mathrm{C}\right)$ for different times. e, $\mathbf{g}$ The EL spectra change under constant voltage of $4.5 \mathrm{~V}$. Lifetime measurement $\left(T_{50}\right)$ of the $(\mathbf{f})$ pristine and $(\mathbf{h}) \mathrm{RbBr}-\mathrm{rich}\langle n\rangle_{\mathrm{RbO} .6}=3 \mathrm{PeLED}$ device at a constant voltage of $4.5 \mathrm{~V}$ 
PL spectra of the pristine $\langle n\rangle_{\mathrm{Rb} 0.6}=3$ perovskite remained unchanged after heating at $100^{\circ} \mathrm{C}$ for $4 \mathrm{~h}$. Similarly, the $\mathrm{RbBr}$ rich $\langle n\rangle_{\mathrm{Rb} 0.6}=3$ perovskite illustrates excellent spectral stability too, in which both the PL peak position and FWHM changed negligibly after $4 \mathrm{~h}$ of annealing (Fig. 5b). In contrast to the previously reported, mixed-halide blue emitter, $\mathrm{PEA}_{2} \mathrm{Cs}_{2} \mathrm{~Pb}_{3}\left(\mathrm{Br}_{0.8} \mathrm{Cl}_{0.2}\right)_{10}$ exhibited poor spectral stability, in which a continuous spectra redshift was observed minutes after annealing began (Fig. 5c). The same phenomena were detected for $\langle n\rangle=2$ quasi-2D perovskite $\mathrm{PEA}_{2} \mathrm{CsPb}_{2} \mathrm{Br}_{7}$ (Fig. $5 \mathrm{~d}$ ), which were in good agreement with the previous reports, indicating that Ostwald ripening probably occurred, which is caused by the Joule heating 35 .

Furthermore, we record the spectral stability of the PeLEDs under continuous operation at a constant voltage of $4.5 \mathrm{~V}$ to extract its lifetime. The half-lifetime $\left(T_{50}\right)$ was defined as the time required for the luminance to decease to $50 \%$ of the start luminance $\left(L_{0}\right)^{11}$. In brief, the EL spectra changes negligibly under continuous operation for $20 \mathrm{~min}$ (Fig. 5e, g) ${ }^{22-24,27}$. The $T_{50}$ for pristine and RbBr-rich $\langle n\rangle_{\mathrm{Rb} 0.6}=3$ PeLEDs are determined to be 5.2 and $14.5 \mathrm{~min}$, respectively (Fig. $5 \mathrm{f}, \mathrm{h}$ ). The result represents the most stable blue PeLEDs reported so far (Supplementary Table 4), which is comparable with the reported stability of green and red analogs $6,8,20,45-50$.

\section{Discussion}

As mentioned above, the ratio of $\mathrm{Rb}$ to $\mathrm{Cs}$, thickness of the quantum wells ( $<n>$ values), as well as content of excess $\mathrm{RbBr}$ synergistically determined the optical properties of the resulting perovskite, including emission wavelength, PLQYs, and spectral stability. Keeping these in mind, by tuning the variable composition, we are able to achieve highly emissive perovskite emitters in the entire blue spectral region (from 454 to $492 \mathrm{~nm}$ ) (Supplementary Fig. 28 and Supplementary Note 4). In particular, the $\mathrm{PEA}_{2}\left(\mathrm{Rb}_{0.8} \mathrm{Cs}_{0.2}\right) \mathrm{Pb}_{3} \mathrm{Br}_{10} \quad\left(\mathrm{RbBr}-\right.$ rich $\left.\quad<n>_{\mathrm{Rb} 0.8}=3\right)$ and $\mathrm{PEA}_{2}\left(\mathrm{Rb}_{0.4} \mathrm{Cs}_{0.6}\right) \mathrm{Pb}_{3} \mathrm{Br}_{10}\left(\mathrm{RbBr}-\right.$ rich $\left.<n>_{\mathrm{Rb} 0.4}=3\right)$ exhibit PLQYs of $58.3 \%$ and $84.1 \%$ after $\mathrm{RbBr}$ treatment, with an emission at $458 \mathrm{~nm}$ (deep blue) and $490 \mathrm{~nm}$ (sky blue), respectively (Supplementary Figs. 20, 28). Meanwhile, we tracked PLQY for RbBrrich $\langle n\rangle_{\mathrm{Rb} 0.8}=3$ and $\mathrm{RbBr}$-rich $\langle n\rangle_{\mathrm{Rb} 0.4}=3$ under ambient condition. As expected, the materials' stability significantly reduced when the ratio of $\mathrm{Rb}$ to Cs was increased (Supplementary Fig. 30), which is consistent with our previous hypothesis. Furthermore, we also fabricated a spectra-stable sky-blue PeLED through the same technique to demonstrate its versatile application. The sky-blue PeLEDs exhibited a peak EQE of $1.48 \%$, peak luminance of $854.3 \mathrm{~cd} \mathrm{~m}^{-2}$, and $T_{50}$ lifetime of $18.7 \mathrm{~min}$ without systematic optimization, representing one of the most stable skyblue PeLEDs (Supplementary Figs. 31, 32, Supplementary Table 4, and Supplementary Note 5).

In summary, we developed an approach to obtain spectra-stable blue-emitting perovskite films with a relatively high PLQY. The approach focuses on engineering the 'A-site' cation to obtain blue emission, which is confirmed to be able to generate stable blue perovskite emitters. We herein designed a $\mathrm{Rb}-\mathrm{Cs}$ alloyed, quasi$2 \mathrm{D}\langle n\rangle_{\mathrm{Rb} 0.6}=3$ perovskite and demonstrated its potential for pure-blue LED application. Composition engineering and in situ passivation are then conducted to regulate its emission property. We achieved a prominent film PLQY of around $82 \%$ for our $\mathrm{RbBr}$-rich $\langle n\rangle_{\mathrm{Rb} 0.6}=3$ perovskite under low excitation density. Encouraged by these findings, we finally achieved a spectra-stable pure-blue PeLED with the peak EQE of $1.35 \%$ and a lifetime of $14.5 \mathrm{~min}$. These materials have overcome the previous problems suffered by the whole community, representing the most efficient and stable blue PeLEDs reported so far. This concept is also demonstrated to be useful in the whole blue emission region and expected to open an avenue for the PeLED community.

\section{Methods}

Materials. PEDOT:PSS (Clevios P VP AL 4083) was purchased from Heraeus. $\mathrm{RbBr}$ (99.6\%), CsBr (99.999\%), and $\mathrm{PbBr}_{2}$ (99.999\%) were bought from SigmaAldrich. PEABr (99.999\%) was purchased from Greatcell Solar. TmPyPB (99\%) and $\mathrm{LiF}$ (99.99\%) were purchased from Lumtech Corp. All of the reagents were directly used without any purification.

LED device fabrication. The precursor solutions were filtered by $0.45-\mu \mathrm{m}$ poly (tetrafluroethylene) filters before using. ITO substrates $\left(10 \Omega \mathrm{sq}^{-1}\right)$ were cleaned by standard procedures and then treated with $\mathrm{O}_{2}$ plasma for $15 \mathrm{~min}$. PEDOT:PSS was then spin-coated on the substrate at 5000 r.p.m. for $30 \mathrm{~s}$, followed by annealing at $150^{\circ} \mathrm{C}$ for $20 \mathrm{~min}$. Then, the substrates were transferred to a nitrogen-filled glovebox. Different DMSO precursor solutions were coated on top of the PEDOT:PSS film via a two-step spin-coating process at 1000 and 8000 r.p.m. for 10 and 30 s, respectively. In the course of the second step, $200 \mu \mathrm{L}$ of chloroform was dropped onto the film, followed by annealing at $100^{\circ} \mathrm{C}$ for another $5 \mathrm{~min}$. Finally, TmPyPB $(40 \mathrm{~nm}), \mathrm{LiF}$ $(0.8 \mathrm{~nm})$, and $\mathrm{Al}$ electrode $(100 \mathrm{~nm})$ were contacted by thermal evaporation. Perovskite precursor solutions were prepared according to Supplementary Table 1.

Device measurements and film characterization. All the PeLEDs were measured in a glovebox. A Keithley 2400 source meter coupled with a fiber integration sphere (FOIS-1-FL, QE65 Pro spectrometer) were used. PeLEDs were measured with a scanning rate of $0.1 \mathrm{~V} \mathrm{~s}^{-1}$ and a dwell time of $1 \mathrm{~s}$. The device active area was determined to be $8.57 \mathrm{~mm}^{2}$. The whole measurement method is consistent with the previous reports ${ }^{12,51}$. The performance of PeLEDs was double-checked using a spectroradiometer of PR-750 to ensure the accuracy and precision of the measurements. The device stability tests were conducted in a glovebox. The EL spectra were collected every $5 \mathrm{~s}$. UV-vis adsorption was recorded from LAMBDA 950 UV/ Vis/NIR spectrophotometer. XRD patterns were obtained using Bruker D8 diffractometer with $\mathrm{Cu} \mathrm{Ka}$ radiation $(\lambda=1.5406 \AA)$. The morphologies of perovskite films were recorded by field-emission SEM (JSM-7500F, JEOL). AFM measurements were collected in noncontact mode (Dimension Icon, Bruker). GIWAXS pattern was measured on beamline 1W2A at Beijing Synchrotron Radiation Facility (BSRF), China. A monochromatic beam of $\lambda=1.54 \AA$ was used, and the incident angle was $0.15^{\circ}$. The fs-TA measurements were carried out on a Helios pump-probe system (Ultrafast Systems LLC) coupled with an amplified femtosecond laser system (Coherent, $35 \mathrm{fs}, 1 \mathrm{kHz}, 800 \mathrm{~nm}$ ). The probe pulses (from 380 to $600 \mathrm{~nm}$ ) were generated by focusing a small portion (around $10 \mu \mathrm{J}$ ) of the fundamental $800-\mathrm{nm}$ laser pulses into a $1-\mathrm{mm} \mathrm{CaF}_{2}$. The $365-\mathrm{nm}$ pump pulses were generated from an optical parametric amplifier (TOPAS-800-fs). The steady-state PL of the perovskite films was measured by a fluorescence spectrophotometer (Fluoromax 4, Horiba) with an excitation wavelength of $365 \mathrm{~nm}$ provided by a 450 $\mathrm{W}$ xenon lamp. The TRPL decay lifetimes were acquired via a monochromator/ spectrograph (Omni- $\lambda 300$, Zolix) and an oscilloscope (GDS-3354, GWINSTEK). The 365-nm pump pulses that are used in the TRPL experiments were the same as those used in the broadband femtosecond TA measurements. The PLQY was measured by a Quanta-Phi integrating sphere, which was an optical fiber bundle coupled with a Fluorolog system, and the excitation wavelength was $365 \mathrm{~nm}$, with an excitation intensity of $1.5 \mathrm{~mW} \mathrm{~cm}^{-2}$. The UPS spectra were obtained on Thermo Scientific ESCALab 250Xi.

\section{Data availability}

The data that support the findings of this study are available from the corresponding author upon reasonable request.

Received: 25 December 2018 Accepted: 2 April 2019

Published online: 23 April 2019

\section{References}

1. Cho, H. et al. Overcoming the electroluminescence efficiency limitations of perovskite light-emitting diodes. Science 350, 1222-1225 (2015).

2. Yuan, M. et al. Perovskite energy funnels for efficient light-emitting diodes. Nat. Nanotechnol. 11, 872-877 (2016).

3. Wang, N. et al. Perovskite light-emitting diodes based on solution-processed self-organized multiple quantum wells. Nat. Photonics 10, 699-704 (2016).

4. Tan, Z. K. et al. Bright light-emitting diodes based on organometal halide perovskite. Nat. Nanotechnol. 9, 687-692 (2014).

5. Ban, M. et al. Solution-processed perovskite light emitting diodes with efficiency exceeding $15 \%$ through additive-controlled nanostructure tailoring. Nat. Commun. 9, 3892 (2018).

6. Zou, W. et al. Minimising efficiency roll-off in high-brightness perovskite light-emitting diodes. Nat. Commun. 9, 608 (2018). 
7. Yang, X. et al. Efficient green light-emitting diodes based on quasi-twodimensional composition and phase engineered perovskite with surface passivation. Nat. Commun. 9, 570 (2018).

8. Zhang, L. et al. Ultra-bright and highly efficient inorganic based perovskite light-emitting diodes. Nat. Commun. 8, 15640 (2017).

9. Huang, $\mathrm{H}$. et al. Growth mechanism of strongly emitting $\mathrm{CH}_{3} \mathrm{NH}_{3} \mathrm{PbBr}_{3}$ perovskite nanocrystals with a tunable bandgap. Nat. Commun. 8, 996 (2017).

10. Wang, H. et al. Trifluoroacetate induced small-grained $\mathrm{Cs} \mathrm{PbBr}_{3}$ perovskite films result in efficient and stable light- emitting devices. Nat. Commun. 10, 665 (2019)

11. Lin, K. et al. Perovskite light-emitting diodes with external quantum efficiency exceeding 20 per cent. Nature 562, 245-248 (2018).

12. Cao, Y. et al. Perovskite light-emitting diodes based on spontaneously formed submicrometre-scale structures. Nature 562, 249-253 (2018).

13. Zhao, B. et al. High-efficiency perovskite-polymer bulk heterostructure lightemitting diodes. Nat. Photonics 12, 783-789 (2018).

14. Jameson, D. \& Hurvich, L. M. Some quantitative aspects of an opponentcolors theory. I. Chromatic responses and spectral saturation. JOSA 45 , 546-552 (1955).

15. Hardin, C. L. \& Maffi, L. Color Categories in Thought and Language (Cambridge University Press, Cambridge, 1997).

16. Kumar, S. et al. Efficient blue electroluminescence using quantum-confined two-dimensional perovskites. ACS Nano 10, 9720-9729 (2016).

17. $\mathrm{Li}, \mathrm{X}$. et al. $\mathrm{CsPbX}$ quantum dots for lighting and displays: room-temperature synthesis, photoluminescence superiorities, underlying origins and white light-emitting diodes. Adv. Funct. Mater. 26, 2435-2445 (2016).

18. Xing, J. et al. Color-stable highly luminescent sky-blue perovskite lightemitting diodes. Nat. Commun. 9, 3541 (2018).

19. Quan, L. N., García de Arquer, F. P., Sabatini, R. P. \& Sargent, E. H. Perovskites for light emission. Adv. Mater. 30, 1801996 (2018).

20. Vashishtha, P. \& Halpert, J. E. Field-driven ion migration and color instability in red-emitting mixed halide perovskite nanocrystal light-emitting diodes. Chem. Mater. 29, 5965-5973 (2017).

21. Wu, Y., Li, X. \& Zeng, H. Highly luminescent and stable halide perovskite nanocrystals. ACS Energy Lett. 4, 673-681 (2019).

22. Li, G. et al. Highly efficient perovskite nanocrystal light-emitting diodes enabled by a universal crosslinking method. Adv. Mater. 28, 3528-3534 (2016).

23. Yao, E. P. et al. High-brightness blue and white LEDs based on inorganic perovskite nanocrystals and their composites. Adv. Mater. 29, 1606895 (2017).

24. Gangishetty, M. K., Hou, S., Quan, Q. \& Congreve, D. N. Reducing architecture limitations for efficient blue perovskite light-emitting diodes. Adv. Mater. 30, 1706226 (2018).

25. Song, J. et al. Quantum dot light-emitting diodes based on inorganic perovskite cesium lead halides $\left(\mathrm{CsPbX}_{3}\right)$. Adv. Mater. 27, 7162-7167 (2015).

26. Pan, J. et al. Highly efficient perovskite-quantum-dot light-emitting diodes by surface engineering. Adv. Mater. 28, 8718-8725 (2016).

27. Hou, S., Gangishetty, M. K., Quan, Q. \& Congreve, D. N. Efficient blue and white perovskite light-emitting diodes via manganese doping. Joule 2, 2421-2433 (2018).

28. Hoke, E. T. et al. Reversible photo-induced trap formation in mixed-halide hybrid perovsktes for photovoltaics. Chem. Sci. 6, 613-617 (2015).

29. Draguta, S. et al. Rationalizing the light-induced phase separation of mixed halide organic-inorganic perovskites. Nat. Commun. 8, 200 (2017).

30. Chen, Z. et al. High-performance color-tunable perovskite light emitting devices through structural modulation from bulk to layered film. Adv. Mater. 29, 1603157 (2017).

31. Congreve, D. N. et al. Tunable light-emitting diodes utilizing quantumconfined layered perovskite emitters. ACS Photonics 4, 476-481 (2017).

32. Xiao, Z. et al. Efficient perovskite light-emitting diodes featuring nanometresized crystallites. Nat. Photonics 11, 108-115 (2017).

33. Wang, Q., Ren, J., Peng, X. F., Ji, X. X. \& Yang, X. H. Efficient sky-blue perovskite light-emitting devices based on ethylammonium bromide induced layered perovskites. ACS Appl. Mater. Int. 9, 29901-29906 (2017).

34. Yang, D. et al. Large-scale synthesis of ultrathin cesium lead bromide perovskite nanoplates with precisely tunable dimensions and their application in blue light-emitting diodes. Nano Energy 47, 235-242 (2018).

35. $\mathrm{Wu}, \mathrm{Y}$. et al. In situ passivation of $\mathrm{PbBr}_{6}{ }^{4-}$ octahedra toward blue luminescent $\mathrm{CsPbr}_{3}$ nanoplatelets with near $100 \%$ absolute quantum yield. ACS Energy Lett. 3, 2030-2037 (2018).

36. Saliba, M. et al. Incorporation of rubidium cations into perovskite solar cells improves photovoltaic performance. Science 354, 206-209 (2016).

37. Linaburg, M. R., McClure, E. T., Majher, J. D. \& Woodward, P. M. Cs $s_{1-x} \mathrm{Rb}_{x} \mathrm{PbCl}_{3}$ and $\mathrm{Cs}_{1-x} \mathrm{Rb}_{x} \mathrm{PbBr}_{3}$ solid solutions: understanding octahedral tilting in lead halide perovskites. Chem. Mater. 29, 3507-3514 (2017).

38. Quan, L. N. et al. Ligand-stabilized reduced-dimensionality perovskites. J. Am. Chem. Soc. 138, 2649-2655 (2016).

39. Byun, J. et al. Efficient visible quasi-2D perovskite light-emitting diodes. $A d v$. Mater. 28, 7515-7520 (2016).
40. Jiang, Y. et al. Reduced-dimensional $\alpha-\mathrm{CsPbX}$ perovskites for efficient and stable photovoltaics. Joule 2, 1215-1216 (2018).

41. Quan, L. N. et al. Tailoring the energy landscape in quasi-2D halide perovskites enables efficient green-light emission. Nano Lett. 17, 3701-3709 (2017).

42. Xing, G. et al. Transcending the slow bimolecular recombination in leadhalide perovskites for electroluminescence. Nat. Commun. 8, 14558 (2017).

43. Li, M. et al. Amplified spontaneous emission based on 2D Ruddlesden-Popper perovskites. Adv. Funct. Mater. 28, 1707006-1707009 (2018).

44. Shi, D. et al. Low trap-state density and long carrier diffusion in organolead trihalide perovskite single crystals. Science 347, 519-522 (2015).

45. Lee, J. W. et al. In-situ formed type I nanocrystalline perovskite film for highly efficient light-emitting diode. ACS Nano 11, 3311-3319 (2017).

46. Wang, J. et al. All-solution-processed pure formamidinium-based perovskite light-emitting diodes. Adv. Mater. 30, 1804137 (2018).

47. Chin, X. Y. et al. Self-assembled hierarchical nanostructured perovskites enable highly efficient LEDs via an energy cascade. Energy Environ. Sci. 11, 1770-1778 (2018)

48. Veldhuis, S. A. et al. Crown ethers enable room-temperature synthesis of $\mathrm{CsPbBr}_{3}$ quantum dots for light-emitting diodes. ACS Energy Lett. 3, 526-531 (2018)

49. Zhang, X. et al. Enhancing the brightness of cesium lead halide perovskite nanocrystal based green light-emitting devices through the interface engineering with perfluorinated ionomer. Nano Lett. 16, 1415-1420 (2016).

50. Yuan, S. et al. Self-assembled high quality $\mathrm{CsPbBr}_{3}$ quantum dot films toward highly efficient light-emitting diodes. ACS Nano 12, 9541-9548 (2018).

51. Forrest, S. R., Bradley, D. D. C. \& Thompson, M. E. Measuring the effeiciency of organic light-emitting devices. Adv. Mater. 15, 1043-1048 (2003)

\section{Acknowledgements}

The authors acknowledge financial support from the National Natural Science Foundation of China (No. 21771114, 11804084), MOE 111 (B12015), the Natural Science Foundation of Tianjin (17JCYBJC40900, 18YFZCGX00580), the Advanced Technology Research Program of Henan Province (182102210369), and the Fundamental Research Funds for the Central Universities. M.Y. acknowledges financial support from Thousand Youth Talents Plan of China.

\section{Author contributions}

M.Y. conceived the idea. J.C. and M.Y. supervised the work. M.Y., G.K., Y.J., Y.H., M.L., L.Z., and J.W. fabricated devices and analyzed the data. C.Q. and M.C. performed TA and TRPL measurements and analyzed the data. T.H., K.L., S.L., and Z.L. performed SEM measurements. H.W. and H.X. performed GIWAXS measurements. Y.J. and M.Y. cowrote the paper. All authors read and commented on the paper.

\section{Additional information}

Supplementary Information accompanies this paper at https://doi.org/10.1038/s41467 019-09794-7.

Competing interests: The authors declare no competing interests.

Reprints and permission information is available online at http://npg.nature.com/ reprintsandpermissions/

Journal peer review information: Nature Communications thanks Qihua Xiong, Xuyong Yang and other anonymous reviewer(s) for their contribution to the peer review of this work. Peer reviewer reports are available.

Publisher's note: Springer Nature remains neutral with regard to jurisdictional claims in published maps and institutional affiliations.

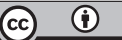

Open Access This article is licensed under a Creative Commons Attribution 4.0 International License, which permits use, sharing, adaptation, distribution and reproduction in any medium or format, as long as you give appropriate credit to the original author(s) and the source, provide a link to the Creative Commons license, and indicate if changes were made. The images or other third party material in this article are included in the article's Creative Commons license, unless indicated otherwise in a credit line to the material. If material is not included in the article's Creative Commons license and your intended use is not permitted by statutory regulation or exceeds the permitted use, you will need to obtain permission directly from the copyright holder. To view a copy of this license, visit http://creativecommons.org/ licenses/by/4.0/.

(c) The Author(s) 2019 